\title{
Do que eles precisam para serem felizes? A felicidade na visão de adolescentes
}

\author{
Síglia Pimentel Höher Camargo \\ Josiane Lieberknecht Wathier Abaid \\ Claudia Hofheinz Giacomoni
}

\begin{abstract}
Resumo
Este trabalho visa investigar o conceito de felicidade para adolescentes. Foi adaptado o roteiro de entrevista semiestruturada sobre bem-estar subjetivo de crianças elaborado por Giacomoni (2002). Participaram do estudo 95 adolescentes entre 12 e 20 anos, estudantes de três escolas públicas do interior do Rio Grande do Sul. As entrevistas transcritas foram submetidas à análise de conteúdo, a partir da qual foram extraídas categorias temáticas. As categorias obtidas indicam uma tendência dos adolescentes em relacionarem sentimentos abstratos e necessidades concretas ao conceito de felicidade. A análise das diferenças significativas por sexo aponta que as meninas relacionaram a felicidade a variadas formas de relacionamento, enquanto os meninos relacionaram-na aos bens materiais e às condições de vida. Esses resultados poderão contribuir na elaboração de instrumentos de avaliação do bem-estar subjetivo em adolescentes e na orientação de intervenções junto a essa faixa etária. Palavras-chave: Psicologia positiva, felicidade, adolescentes.
\end{abstract}

\section{What do they need to be happy? The happiness for adolescents}

\begin{abstract}
In this paper we aim at investigating the concept of happiness from the adolescents' perspective. For this purpose we adapted the partially structured interview script about children's subjective well being produced by Giacomoni (2002). We studied 95 adolescents, between 12 and 20 years, students of tree public schools of the interior of the Rio Grande do Sul. The transcribing interviews were submitted to a content analysis, from which we extracted thematic categories. The categories we got from the study pointed to a trend of the adolescents in mentioning abstract feelings and concrete necessities related to happiness. The analysis of significant differences by sex pointed that girls mentioned diverse kinds of relationships related to happiness, while boys tend to correlate happiness with personal belonging and life conditions. These findings can contribute to the construction of subjective well-being assessment scales for teenagers. Moreover, they can help educator in giving orientation and making intervention when necessary.
\end{abstract}

Key words: Positive psychology, happiness, adolescents.

\section{Qué necesitan para ser felices? La felicidad según los adolescentes}

\section{Resumen}

Este trabajo busca investigar el concepto de felicidad para adolescentes. Se adaptó el cuestionario de entrevista semi-estructurada sobre bienestar subjetivo de niños elaborado por Giacomoni (2002). Participaron del estudio 95 adolescentes entre 12 y 20 años, estudiantes de tres escuelas públicas del interior de Rio Grande do Sul. Se extrajeron categorías temáticas a partir del análisis de contenido de las entrevistas. Las categorías levantadas indican tendencia de los adolescentes a relacionar sentimientos abstractos y necesidades concretas al concepto de felicidad. El análisis de las diferencias significativas por sexo señala que las niñas relacionaron la felicidad a variadas formas de relacionamiento, mientras que los niños la relacionaron a bienes materiales y a condiciones de vida. Estos resultados podrán contribuir en la elaboración de instrumentos de evaluación del bienestar subjetivo de adolescentes y en la orientación de intervenciones junto a esta franja de edad.

Palabras clave: Psicología positiva, felicidad, adolescentes. 


\section{Introdução}

Alvo de estudos da Filosofia em diferentes épocas (Lastória, 2003), a felicidade, também conhecida pelo termo científico "bem-estar subjetivo", passou a ser mais amplamente estudada com o surgimento de uma nova perspectiva no campo da Psicologia, preconizada por Seligman e Csikszentmihalyi (2000): a Psicologia Positiva. Essa perspectiva propõe uma mudança de foco de uma ciência que, desde a Segunda Guerra Mundial, tem priorizado os processos psicopatológicos e suas implicações, seguindo um modelo médico de doença. A Psicologia Positiva visa incentivar os psicólogos a adotarem uma postura mais aberta e apreciativa dos potenciais humanos, das motivações e das capacidades que, no nível subjetivo, procura abarcar o estudo da felicidade (Sheldon \& King, 2001).

Sendo assim, o bem-estar subjetivo refere-se ao que as pessoas pensam e como elas se sentem em relação as suas vidas, o que, inevitavelmente, envolve uma avaliação subjetiva da qualidade de vida. Esta avaliação da pessoa sobre as suas vivências pode ser feita de forma cognitiva, quando faz um julgamento consciente dos diversos domínios como trabalho, lazer e relacionamentos. Também pode haver uma avaliação de suas emoções e humores prazerosos ou desprazerosos experienciados. Trata-se, portanto, de uma avaliação tanto cognitiva quanto emocional da própria existência (Diener, 1984; Diener, Suh, \& Oishi, 1997; Diener, Suh, Lucas, \& Smith, 1999). Neste sentido, se uma pessoa apresenta alto nível de bem-estar subjetivo, significa que ela está experienciando satisfação de vida frequente, emoções de contentamento, alegria e infrequentes emoções como raiva e tristeza que, quando frequentes, não indicam altos níveis de bem-estar subjetivo (Giacomoni, 2002; Giacomoni \& Hutz, 2008).

O bem-estar subjetivo é, assim, uma área que tem crescido reconhecidamente nos últimos tempos. Cabe ressaltar, entretanto, uma noção importante de que bem-estar subjetivo não é sinônimo de saúde mental ou psicológica. Uma pessoa pode estar feliz e satisfeita com sua vida mesmo em estado de delírio, o que não se autoriza afirmar que ela possua saúde mental. Assim, o bem-estar subjetivo não é uma condição suficiente para o bem-estar psicológico, mas é uma condição desejável (Diener e cols., 1997). O bem-estar subjetivo volta-se para os fatores que diferenciam as pessoas felizes em diferentes graus (feliz, moderadamente feliz ou extremamente feliz) e é definido a partir da experiência interna do respondente, ou seja, é avaliado a partir da perspectiva do próprio indivíduo (Diener e cols., 1997; Dell'Aglio, Koller, \& Yunes, 2006). Suas crenças sobre seu próprio bem-estar são de fundamental importância para esta avaliação. Segundo Diener e Lucas (2000), as pessoas avaliam as condições de vida de forma diferente, dependendo das suas expectativas, valores e experiências prévias. Além disso, o bem-estar subjetivo focaliza também os estados de longo prazo e não somente humores momentâneos, ainda que estejam sujeitos a alterações conforme novos eventos venham a acontecer (Diener e cols., 1997). Relacionado a isso, sob o ponto de vista desenvolvimental, de qualidade de vida e resiliência, sabe-se que a proximidade com os irmãos pode propiciar apoio emocional, estendendo-se, na vida adulta, em maior competência social e iniciativa no auxílio ao outro. A existência de cuidado entre irmãos tem sido apontada pela literatura (Luthar \& Zigler, 1991; Werner \& Smith, 1992, 1998) como fator auxiliar no processo de socialização da criança, especialmente de meninas com relação aos irmãos menores.

Atualmente, encontramos alguns estudos sobre o bem-estar subjetivo de crianças e adolescentes na literatura brasileira. Assumpção Jr., Kuczynski, Sprovieri e Aranha (2000) realizaram um estudo de validação de uma escala de avaliação da qualidade de vida em crianças. A escala leva em consideração o contexto pediátrico e o desenvolvimento da criança, e as suas qualidades psicométricas permitem o seu uso como um importante instrumento de avaliação diagnóstica. Outro estudo, realizado por Giacomoni (2000) com 200 crianças entre 5 e 12 anos, investigou o desenvolvimento do conceito de felicidade e suas principais características em crianças de idade escolar. Foram geradas sete categorias relativas aos domínios de satisfação de vida infantil (Self, Família, Amizade, Escola, Satisfação de Necessidades Básicas, Satisfação de Desejos, Lazer e Não Violência) a partir das quais foi possível desenvolver um instrumento de avaliação do bem-estar subjetivo infantil (Giacomoni \& Hutz, 2008). Arteche e Bandeira (2003) realizaram um estudo sobre o bem-estar subjetivo de 193 adolescentes trabalhadores de 14 a 17 anos em relação ao trabalho. Os resultados indicaram bons níveis de bem-estar e mostraram que o trabalho na adolescência pode ser positivo, sobretudo para os adolescentes que realizam um trabalho educativo, ou seja, uma modalidade de trabalho em que as necessidades pedagógicas superam as questões produtivas (Estatuto da Criança e do Adolescente, 1990). Em outro estudo com adolescentes universitários, Dela Coleta e Dela Coleta (2006) mostraram que estes estudantes avaliam sua satisfação, bem-estar e felicidade de modo positivo.

Pode-se perceber, portanto, que, apesar da reconhecida relevância dos estudos nacionais já realizados no campo do bem-estar subjetivo, estes ainda são escassos, principalmente no que se refere à adolescência e à investigação da noção subjetiva que os adolescentes possuem sobre o que venha a ser felicidade. Diante disso, percebe-se a importância da realização de pesquisas a partir da visão dos próprios adolescentes, as quais podem viabilizar o desenvolvimento de programas de intervenção mais efetivos junto aos mesmos. Considerando estes aspectos, o presente estudo teve por meta investigar e analisar o conceito de felicidade descrito pelos adolescentes. Também teve como objetivo verificar possíveis diferenças entre os sexos e a série no que se refere a esse construto. 


\section{Método}

\section{Participantes}

Participaram deste estudo 95 adolescentes, de ambos os sexos, sendo 48 meninas (51\%) e 47 meninos (49\%), estudantes de escolas públicas da cidade de Santa Maria-RS. A idade variou entre 12 e 20 anos ( $M=14,3$; $D P=1,3)$. $\mathrm{O}$ número de irmãos referido variou de zero a oito, sendo o número médio encontrado de 1,7 irmãos $(\mathrm{DP}=1,4)$. Todos os participantes frequentavam o ensino fundamental, sendo que 37 estavam matriculados na sétima série e 58 , na oitava série.

\section{Instrumentos}

Para a realização desta pesquisa, foi utilizada a técnica de entrevistas individuais, a partir de um roteiro de entrevista semiestruturada. Este roteiro, composto por 14 questões, consistiu numa versão adaptada do roteiro de entrevista semiestruturada para crianças desenvolvido por Giacomoni (2002). Nos primeiros itens do roteiro, encontraram-se os dados de identificação dos entrevistados, tais como: iniciais do nome, sexo, idade e número de irmãos. Para adaptação do roteiro da entrevista, foram realizados dois estudos pilotos. Ambos tiveram como objetivo avaliar a compreensão das questões por parte dos adolescentes e a validade do instrumento. No entanto, este artigo enfatiza os achados relacionados às análises das duas primeiras questões, uma vez que estas exploraram o conceito de felicidade atribuído pelos adolescentes.

\section{Procedimentos}

Para a realização das entrevistas, foi solicitada à $8^{a}$ Coordenadoria Regional de Educação do RS uma listagem de todas as escolas públicas e privadas da cidade de Santa Maria e região, uma vez que a escola apresenta-se como um meio de contato direto com adolescentes. Foram selecionadas, por conveniência, três escolas públicas de ensino fundamental da cidade de Santa Maria/ RS, adotando como critério a proximidade do estabelecimento e facilidade de acesso às pesquisadoras. Entrou-se em contato com a direção e coordenação pedagógica da escola, quando foi apresentada a pesquisa, explicitando seus objetivos e procedimentos. Obtida a autorização da direção da escola e dos professores, após a apresentação da proposta de trabaIho, foi realizado um encontro com os alunos. O pesquisador teve o contato inicial com os adolescentes na própria sala de aula, explicando de forma coletiva o procedimento do estudo e sua finalidade, deixando claro o caráter voluntário de sua participação. A entrevista foi realizada individualmente, mediante o assentimento do aluno, que já portava o consentimento de um dos seus responsáveis legais. Todas as entrevistas foram gravadas em fitas $\mathrm{K} 7$ e depois transcritas na íntegra. As entrevistas que apresentaram problemas em alguma das fases descritas foram descartadas.

\section{Considerações Éticas}

Considerando as questões éticas que devem ser observadas nas pesquisas envolvendo seres humanos, o presente estudo cumpre com as exigências, normas e orientações previstas pelo Conselho Nacional de Saúde (Resolução n. 196/96, 1996) e pelo Conselho Federal de Psicologia (Resolução n. 016/2000). Desse modo, garantiu-se, a todos os participantes, o direito de sigilo, a participação voluntária e a interrupção da participação sem prejuízo ao participante. A entrevista foi realizada individualmente em local fornecido pela escola e gravada mediante autorização do participante.

\section{Análise de dados}

Após a transcrição das entrevistas, as respostas obtidas para cada questão foram submetidas a uma análise de conteúdo, segundo Bardin (1979/2004). A análise permitiu identificar categorias temáticas a posteriori entre as respostas fornecidas pelos adolescentes. O processo de identificação de categorias temáticas foi realizado separadamente $e$ simultaneamente por duas equipes de pesquisadoras, que, posteriormente, desenvolveram, em conjunto, um manual de definições das categorias extraídas. Foi realizada uma análise estatística descritiva para levantar as frequências e os percentuais das categorias encontradas no grupo todo, bem como separadas por sexo e por série.

\section{Resultados}

Com o intuito de verificar o conceito de felicidade na visão dos adolescentes, foram analisadas as respostas referentes às seguintes questões: "O que vem na tua cabeça quando tu pensas em felicidade?" (Questão 1) e "O que é estar feliz?" (Questão 2). Para cada questão, foram elencadas categorias temáticas, seguidas de suas respectivas definições, as quais partiram dos critérios de exclusão, recorrência do conteúdo, pertinência e homogeneidade, ou seja, as definições deveriam ser claras o suficiente para que um item pertencesse a apenas uma categoria, conforme recomendado por Bardin (1979/2004). Foram contabilizados os itens de resposta mencionados mais de uma vez por entrevistado em relação a cada pergunta.

A primeira questão teve por objetivo chamar o respondente ao tema proposto e, sendo introdutória, registrar as primeiras impressões ao ouvir a palavra "felicidade". Foram extraídas nove categorias temáticas, as quais são apresentadas a seguir. A concordância entre juízes foi superior a $76 \%$, o que indica que as categorias foram formuladas e descritas de modo claro e metodologicamente satisfatório. 
1. FAMÍLIA: Refere-se à instituição família, incluindo seus membros, eventos, relacionamentos, ações e sentimentos característicos. Ex.: "Estar com a família, pais, quando eu casar".

2. SENTIMENTOS: Refere-se a entidades consideradas abstratas ao descrever os sentimentos relacionados à felicidade, incluindo sentimentos altruístas e de autonomia, tais como: "paz, carinho, liberdade, vida", dentre outros.

3. AMIZADE: Inclui ações, sentimentos e o relacionamento entre amigos, além da mera referência a esses, incluindo inimizades. Assim sendo: "Estar com meus amigos, sair com amigos" fazem parte desta categoria.

4. AUTORREFERENNCIA: Inclui referências ao eu e suas pretensões. Dessa forma, incluem-se nesta categoria: "em mim", "meu futuro", dentre outros.

5. ATIVIDADES / LAZER: Refere-se a ações diversas realizadas pelos sujeitos, as quais Ihes trazem felicidade. Como exemplos, temos: "sair" e "praticar esportes".

6. RELACIONAMENTOS: Inclui diferentes formas de relações entre sujeitos (pares e outros), suas implicações, ações em conjunto e a satisfação trazida por essas. Sendo assim: "encontrar as pessoas que a gente gosta", "namorada", "pessoas que gostam da gente" são exemplos.

7. SATISFAÇÃO DAS NECESSIDADES MATERIAIS E DE DESEJO: Inclui situações almejadas e/ou conquista- das pelo sujeito em relação a bens materiais, conquistas futuras e profissionais, bem como referência à saúde. Como exemplos desta categoria, temos: "bens materiais", "morar bem", "segurança" e "condições de vida".

8. ESCOLA: Refere-se a ações, relacionamentos e atividades relacionadas ao ambiente escolar, assim como à satisfação trazida por fazer parte deste. Como exemplos de itens desta categoria, temos: "vir pro colégio", "colegas" e "não ter discussão no colégio".

9. OUTROS: Inclui quaisquer referências que não possam ser incluídas nas demais categorias. Exemplos: "tudo, "Deus", "não sei”, "caixa de bombom”, "rindo", falando".

As Tabelas 1 e 2 apresentam as frequências e percentuais para a amostra total, por sexo e por série, além dos coeficientes indicativos de diferenças.

Considerando a frequência como número de vezes em que um item de resposta foi citado pelos adolescentes na questão 1, pode-se perceber que, dentre os 95 entrevistados, a categoria "Sentimentos" foi a mais lembrada, seguida da categoria "Família". Para avaliar as diferenças por sexo e série, utilizou-se o teste estatístico não paramétrico $\mathrm{U}$ de Wilcoxon-Mann-Withney, que avalia se existe diferença estatística significativa entre as medianas de duas (Dancey, 2006), uma vez que os dados do presente estudo são assimétricos e tem-se um pequeno número de participantes. Em relação aos sexos (ver Tabela 1), a categoria mais frequentemente citada pelos meninos foi "Sentimentos", seguida por "Família" e "Satisfação das Necessidades e Desejos". Já as

Tabela 1. Frequências e percentuais totais e por sexo referentes às categorias da Questão 1.

\begin{tabular}{|c|c|c|c|c|c|c|c|c|c|c|}
\hline \multirow[t]{2}{*}{ Categorias questão 1} & \multirow[b]{2}{*}{$f$} & \multirow[b]{2}{*}{$\%$} & \multicolumn{3}{|c|}{ Meninos } & \multicolumn{3}{|c|}{ Meninas } & \multirow[b]{2}{*}{$U$} & \multirow[b]{2}{*}{$p$} \\
\hline & & & $f$ & $\%$ & Mediana & $f$ & $\%$ & Mediana & & \\
\hline SENTIMENTOS & 70 & 28,3 & 36 & 30,3 & 49,3 & 34 & 26,6 & 46,7 & $1.067,0$ & 0,610 \\
\hline FAMÍLIA & 61 & 24,7 & 24 & 20,2 & 44,7 & 37 & 28,9 & 51,2 & 974,5 & 0,190 \\
\hline $\begin{array}{l}\text { SATISFAÇÃO DAS NEC. } \\
\text { MATERIAIS E DE DESEJO }\end{array}$ & 35 & 14,2 & 23 & 19,3 & 52,5 & 12 & 9,4 & 43,6 & 914,5 & $0,039^{*}$ \\
\hline AMIZADE & 29 & 11,7 & 15 & 12,6 & 46,8 & 14 & 10,9 & 49,2 & $1.071,0$ & 0,570 \\
\hline RELACIONAMENTOS & 19 & 7,7 & 5 & 4,2 & 43,9 & 14 & 10,9 & 51,1 & 935,0 & 0,055 \\
\hline OUTROS & 13 & 5,3 & 5 & 4,2 & 45,7 & 8 & 6,3 & 49,3 & $1.020,0$ & 0,270 \\
\hline ATIVIDADES / LAZER & 11 & 4,5 & 6 & 5,0 & 46,6 & 5 & 3,9 & 46,4 & $1.051,0$ & 0,920 \\
\hline ESCOLA & 5 & 2 & 3 & 2,5 & 48,0 & 2 & 1,6 & 48,0 & $1.126,0$ & 0,960 \\
\hline AUTORREFERÊNCIA & 4 & 1,6 & 2 & 1,7 & 48,0 & 2 & 1,6 & 48,0 & $1.127,0$ & 0,980 \\
\hline Total & 247 & 100 & 119 & 100,0 & & 128 & 100,0 & & & \\
\hline
\end{tabular}

${ }^{*} p<0,05$ 
meninas citaram com maior frequência a categoria "Família", seguida de "Sentimentos". As estatísticas descritivas mostraram que, apesar de o teste $U$ não ter indicado diferenças significativas entre as variáveis medidas na Tabela 1, houve uma tendência das meninas conceituarem a felicidade com mais itens relacionados à categoria "Relacionamentos" do que os meninos. Por outro lado, houve diferença significativa entre os sexos na categoria "Satisfação das Necessidades e Desejo", sendo que os meninos citaram mais itens relacionados a esta categoria do que as meninas. Nas demais categorias, não houve diferença significativa por sexo.

Em relação à escolaridade dos participantes (Tabela 2), os estudantes da sétima série apontaram com maior frequência a categoria "Sentimentos", seguidos da categoria "Família" e "Satisfação das necessidades Materiais e Desejos", e os alunos da oitava série apontaram mais as categorias "Família" e "Sentimentos". O teste U de Mann-Withney apontou a existência de diferença significativa na categoria "Satisfação de Necessidades e Desejo", sendo que os participantes pertencentes à sétima série obtiveram mediana de itens mais alta do que os alunos que estudavam na oitava série. Nas demais categorias, não houve diferença significativa entre as séries.

Em relação à questão 2, "O que é estar feliz?", foram obtidas 8 categorias referentes ao conceito de felicidade. Algumas definições das categorias de conteúdo de respostas para a questão 2 mantiveram-se semelhantes às propostas na questão 1. A seguir, descrevemos apenas a definição das categorias que receberam definições diferentes devido ao seu conteúdo. $O$ índice de concordância entre juízes sobre as categorias foi superior a $75 \%$. Categorias:
1. FAMÍLIA;

\section{RELACIONAMENTOS;}

\section{AMIZADE;}

4. SENTIMENTOS (incluindo itens de autorreferência e de autonomia);

5. ALTRUÍSMO: Engloba as intenções e ações positivas do sujeito em relação aos outros, sem, no entanto, ter expectativa de retorno dessas. Como exemplos, podemos citar: "ver os outros felizes" e "ajudar os outros";

6. ATIVIDADES / LAZER: Inclui ações diversas, realizadas pelos sujeitos, podendo ou não ser prazerosas, na quais se incluem eventos sociais. Como exemplos: "fazer ginástica", "fazer o que gosta".

7. SATISFAÇÃO DAS NECESSIDADES MATERIAIS E DE DESEJO: Refere-se a situações almejadas e/ou conquistadas pelo sujeito em relação a bens materiais e desejos, conquistas futuras, incluindo a não violência, ter saúde, escola, profissão. Dentre as referências a esta categoria, temos: "quando consigo ter coisa boa que queria", "ter casa" e "não ter problemas".

\section{OUTROS.}

Tabela 2. Frequências e percentuais totais e por série referentes às categorias da Questão 1.

\begin{tabular}{|c|c|c|c|c|c|c|c|c|c|c|}
\hline \multirow[t]{2}{*}{ Categorias questão 1} & \multirow[b]{2}{*}{$f$} & \multirow[b]{2}{*}{$\%$} & \multicolumn{3}{|c|}{$7^{a}$ série } & \multicolumn{3}{|c|}{$8^{a}$ série } & \multirow[b]{2}{*}{$U$} & \multirow[b]{2}{*}{$p$} \\
\hline & & & $f$ & $\%$ & Mediana & $f$ & $\%$ & Mediana & & \\
\hline SENTIMENTOS & 70 & 28,3 & 31 & 30,4 & 51,26 & 39 & 26,9 & 45,92 & 952,5 & 0,30 \\
\hline FAMÍLIA & 61 & 24,7 & 21 & 20,6 & 43,89 & 40 & 27,6 & 50,62 & 921,0 & 0,18 \\
\hline $\begin{array}{l}\text { SATISFAÇÃO DAS NEC. } \\
\text { MATERIAIS E DE DESEJO }\end{array}$ & 35 & 14,2 & 21 & 20,6 & 53,73 & 14 & 9,7 & 44,34 & 861,0 & $0,036^{*}$ \\
\hline AMIZADE & 29 & 11,7 & 11 & 10,8 & 48,28 & 18 & 12,4 & 47,82 & 1062,5 & 0,91 \\
\hline RELACIONAMENTOS & 19 & 7,7 & 9 & 8,8 & 49,18 & 10 & 6,9 & 46,41 & 992,5 & 0,47 \\
\hline OUTROS & 13 & 5,3 & 3 & 2,9 & 45,27 & 10 & 6,9 & 48,95 & 972,0 & 0,27 \\
\hline ATIVIDADES / LAZER & 11 & 4,5 & 5 & 4,9 & 47,42 & 6 & 4,1 & 45,88 & 983,5 & 0,58 \\
\hline ESCOLA & 5 & 2 & 0 & 0 & 46,00 & 5 & 3,4 & 49,28 & 999,0 & 0,10 \\
\hline AUTORREFERENCCIA & 4 & 1,6 & 1 & 1 & 47,28 & 3 & 2,1 & 48,46 & 1046,5 & 0,56 \\
\hline Total & 247 & 100 & 102 & 100 & - & 145 & 100 & - & - & - \\
\hline
\end{tabular}

${ }^{*} p<0,05$ 
Tabela 3. Frequências e Percentuais totais e por série referentes às categorias da Questão 2.

\begin{tabular}{|c|c|c|c|c|c|c|c|c|c|c|}
\hline \multirow[t]{2}{*}{ Categorias questão 2} & \multirow[b]{2}{*}{$f$} & \multirow[b]{2}{*}{$\%$} & \multicolumn{3}{|c|}{$7^{a}$ série } & \multicolumn{3}{|c|}{$8^{a}$ série } & \multirow[b]{2}{*}{$U$} & \multirow[b]{2}{*}{$p$} \\
\hline & & & $\mathrm{F}$ & $\%$ & Mediana & $f$ & $\%$ & Mediana & & \\
\hline SENTIMENTOS & 50 & 22,5 & 25 & 26,9 & 52,65 & 25 & 19,4 & 45,03 & 901,0 & 0,136 \\
\hline $\begin{array}{l}\text { SATISFAÇÃO DAS NEC. } \\
\text { MATERIAIS E DE DESEJO }\end{array}$ & 38 & 17,1 & 21 & 22,6 & 54,32 & 17 & 13,2 & 43,97 & 839,0 & $0,026^{*}$ \\
\hline ATIVIDADES / LAZER & 37 & 16,7 & 19 & 20,4 & 48,62 & 18 & 13,9 & 47,60 & 1050,0 & 0,812 \\
\hline RELACIONAMENTOS & 32 & 14,4 & 8 & 8,6 & 42,39 & 24 & 18,6 & 50,82 & 865,5 & $0,067^{* *}$ \\
\hline FAMÍLIA & 23 & 10,4 & 8 & 8,6 & 47,23 & 15 & 11,6 & 48,49 & 1044,5 & 0,74 \\
\hline AMIZADE & 25 & 11,3 & 7 & 7,5 & 48,80 & 18 & 14 & 46,66 & 1006,5 & 0,55 \\
\hline OUTROS & 11 & 4,9 & 4 & 4,3 & 46,95 & 7 & 5,4 & 48,67 & 1034,0 & 0,57 \\
\hline ALTRUÍSMO & 6 & 2,7 & 1 & 1,1 & 46,77 & 5 & 3,9 & 48,78 & 1027,5 & 0,369 \\
\hline Total & 222 & 100 & 93 & 100 & - & 129 & 100 & - & - & - \\
\hline
\end{tabular}

Pode-se perceber que, quanto ao que os adolescentes consideram o que seja estar feliz, obteve-se, com maior frequência, as categorias "Sentimentos, "Satisfação de necessidades materiais e de desejo" e "Atividades/ Lazer". Chama-se a atenção para a categoria com menor frequência: "Altruísmo". Nenhuma das categorias da questão 2 teve diferença significativa por sexo. Na Tabela 3, constam as frequências e percentuais totais e por série quanto às categorias sobre o que é estar feliz.

Foi realizado um teste $U$ de Mann-Wihtney para verificar diferenças entre as categorias por série. Os participantes pertencentes à sétima série obtiveram mediana de itens mais alta na categoria de "Satisfação de Necessidades e Desejo" do que os alunos que estudavam na oitava série. Já os adolescentes que estudavam na oitava série sobressaíram-se na categoria "Relacionamentos". As demais categorias da questão 2 não tiveram diferença significativa por série, como se pode ver na Tabela 3.

\section{Discussão}

A partir das categorias extraídas na questão 1 , pode-se perceber uma tendência inicial dos adolescentes em relacionar o termo "felicidade" com sentimentos, o que demonstra a necessidade do adolescente de se sentir bem consigo mesmo e com os outros em meio às tensões provocadas pelas mudanças físicas e emocionais. Na categoria "Sentimentos", foi possível identificar itens citados pelos adolescentes relacionados à sensação de bem-estar, prazer, aceitação e autonomia. Do mesmo modo, pode-se pensar que a "Família", a segunda categoria mais frequentemente citada, é considerada importante pelos adolescentes por ser esta a primeira rede de apoio do indivíduo, como uma base segura a partir da qual se pode explorar o mundo, sobretudo o mundo do relacionamento com os companheiros. Esse resultado corrobora a literatura especializada, que aponta a família como o núcleo onde as primeiras necessidades são satisfeitas e as experiências socializadoras são vivenciadas (Assis e cols., 2003; Steinberg, 1999). Em um estudo realizado por Zagury (2003), 30\% da amostra composta por 943 adolescentes, com idades entre 14 e 19 anos e pertencentes às cinco diferentes classes sociais, apontaram a família unida como fundamental para a felicidade. No presente estudo, apesar de menos frequente, as categorias "Amizade" e "Relacionamentos" também foram consideradas importantes e influentes no conceito de felicidade atribuído pelos adolescentes. As "Atividades e o lazer" (4,5\%) parecem ter menor relevância, ou seja, não estão primeiramente associados com a noção de felicidade, seguidos pela "Escola" $(2,0 \%)$ e "Autorreferência" (1,6\%). 
Quanto ao fato de a categoria "Família" ter sido mais frequentemente citada entre as meninas, pode-se inferir que elas possuem o núcleo familiar como principal rede de apoio (Dell'Aglio \& Hutz, 2002). Essa influência pode ser consequência da educação e da cultura vivenciadas pelas adolescentes, em que a mulher está mais voltada para os eventos que se realizam no interior da família e os relacionamentos ali envolvidos, embora exista atualmente grande transformação cultural neste sentido (Assumpção Jr., 2008; Malpique, Lima, Soleiro, \& Confraria, 2003; Yunes, 2003). A categoria "Relacionamentos" foi citada pelas adolescentes com uma frequência bastante próxima à da categoria "Família" e, embora sem diferença significativa em relação aos meninos, deve ser considerada como importante sob o ponto de vista psicológico (Dancey, 2006). Esses achados refletem uma característica comum do comportamento mais disponível da adolescente do sexo feminino, ao se preocupar com ter alguém para conversar, dividir as dúvidas e angústias bastante presentes nesta fase da vida. Associa-se a isso o fato de que, durante seu desenvolvimento, as meninas mais rapidamente alcançam a maturação emocional, estando mais atentas do que os meninos aos relacionamentos, sejam esses com amigos ou com namorados (Steinberg, 1999). Segundo Narvaz (2001), o senso feminino de identidade desenvolve-se mais pelo estabelecimento de relacionamentos do que pela conquista de uma identidade individual, o que justificaria o destaque à categoria relacionamentos entre as meninas no que tange ao conceito de felicidade.

Os meninos, ainda que geralmente menos maduros emocionalmente, comparados ao sexo oposto de mesma faixa etária (Steinberg, 1999), apontam os "Sentimentos" $(30,3 \%)$ como a categoria mais lembrada no presente estudo. Culturalmente, eles são menos estimulados a perceber e identificar sentimentos, sejam eles pessoais ou alheios (Steinberg, 1999). Entretanto, tais dados refletem a inadequação de pré-concepções a este respeito, mostrando que adolescentes do sexo masculino também se preocupam com questões emocionais próprias e dos outros. Além disso, incluem-se nessa categoria os sentimentos de autonomia e liberdade, os quais são marcantes nessa etapa do desenvolvimento. A segunda categoria mais citada pelos meninos foi "Família" e "Satisfação de Necessidades e desejo", ambas com a mesma frequência. Isso novamente reforça a importância que é atribuída pelos adolescentes ao suporte familiar em ambos os sexos e o quanto as determinações culturais para o que se espera do sexo masculino podem influenciar a noção da felicidade em meninos. A categoria "Satisfação das Necessidades e desejo", a qual apresentou uma diferença significativa em relação às meninas, associou a felicidade à obtenção de "bens materiais", "morar bem", "segurança", "ter condições de vida". Além disso, a cultura do consumo, que, através da mídia, cria necessidades e estabelece relações de dependência entre bem-estar e aceitação com a obtenção de um produto, pode ter influenciado na emergência desta categoria. Entretanto, a referida categoria também pode ter apresentado uma frequência elevada por abranger as necessidades materiais como uma série de condições sine qua non para a qualidade de vida para a felicidade (morar bem, ter segurança, boas condições de vida). Na literatura, encontra-se que, se as condições básicas de sobreviência são mantidas, o dinheiro não se relaciona com a felicidade (Seligman \& Csikszentmihalyi, 2000; Graziano, 2005).

Em relação à escolaridade, houve diferença significativa apenas na categoria "Satisfação das Necessidades e de Desejo", sendo que os alunos da sétima série consideraram esses fatores como associados ao conceito de felicidade. Isso pode ser explicado pela preocupação dos estudantes da oitava série estar mais centrada nos aspectos desenvolvimentais relacionados à finalização do ensino fundamental, tais como: aprovação no ano letivo, mudanças de escola e separação de antigos colegas e de amigos. Isso coincide com a maior frequência da categoria "Sentimentos" e "Família" estarem associadas à felicidade para os adolescentes da $8^{a}$ série.

A partir das categorias extraídas na segunda questão, observou-se que os adolescentes indicaram que o estar feliz se relaciona a "Sentimentos" positivos vinculados à felicidade, em que se inclui, sobretudo, o bem-estar próprio, como se pode constatar nas verbalizações "estar bem consigo", "gostar de si mesmo", "de bem com a vida", "pensar em coisas boas" etc. Isso demonstra que os adolescentes valorizam e priorizam aspectos subjetivos, sabem reconhecê-los e, em certa medida, os consideram mais relevantes do que outros indicadores de felicidade. Esse resultado também é ressaltado no estudo de Assis e cols. (2003) em que os adolescentes apresentaram uma visão extremamente otimista de si, ao contrário da que os adultos e a sociedade em geral têm sobre eles.

Por outro lado, os participantes não deixaram de citar, como já constatado na questão 1 , que estar feliz também diz respeito a questões de necessidades materiais, como ter uma casa e boas condições de vida, além de necessidades de desejo e conquistas futuras. A ordem de importância das categorias obtidas permite pensar que a primeira (Sentimentos) surge como consequência das demais, portanto, para "ter paz", "carinho" e "estar de bem consigo mesmo", é necessário ter "satisfação de necessidades materiais e de desejo" atendidas, ter "Relacionamentos", "Atividade/lazer", "Família" e "Amizade".

É importante destacar que as categorias "Família" (10\%) e "Amizade" (11,3\%), encontram-se mais distantes das categorias mais mencionadas pelos adolescentes ("Sentimentos", 22,5\%, "Satisfação de necessidades materiais e de desejo", 17,1\% e "Atividades de lazer", 16,7\%). Isso parece espelhar a característica própria da adolescência, de almejar sentir-se bem frente aos vários conflitos encontrados em diversas dimensões de sua vida, tais como mudanças corporais, identidade, limites, independência e grupo de amigos (Ferreira \& Marturano, 2002; Steinberg, 1999), associados a questões de possuir bens materiais ou de obter conquistas, o que, muitas vezes, determina ser valorizado ou aceito, ou ainda desperta o sentimento de pertença a um determinado grupo. Os adolescentes compreenderam, de maneira geral, que estar feliz é uma condição mais situacional do que o 
conceito de felicidade e associaram o estado de estar feliz com prática de lazer e esportes ou necessidades materiais (Passarelli \& Silva, 2007).

Observou-se, ainda, que não houve diferença significativa em relação ao sexo quanto ao que é estar feliz. Pode-se pensar que estar feliz tem um significado semelhante entre adolescentes do sexo feminino ou masculino. A esse respeito, Diener (1996) e também Huebner, Drane e Valois (2000) afirmam que o bem-estar subjetivo manifesta-se independentemente do gênero, classe econômica ou escolaridade do adolescente.

\section{Considerações finais}

Esse artigo teve como objetivo contribuir para a área de estudos sobre a qualidade de vida subjetiva dos adolescentes, buscando identificar os conceitos de bem-estar subjetivo a partir da perspectiva deles. Muitas vezes, a profundidade ou amplitude dos conflitos vivenciados pelos adolescentes em função das novas exigências relativas ao período de desenvolvimento, tal como a proximidade de amadurecimento de relacionamentos amorosos e a escoIha profissional, reflete-se no índice de depressão e queda de autoestima que é observado no início da adolescência (Abaid, Dell'Aglio, \& Koller, 2010; Cole e cols., 2002).

Os resultados aqui apresentados e discutidos podem auxiliar nas ações em diversos contextos em que vive o adolescente, sobretudo no ambiente escolar, ocupacional, existencial e clínico. É necessário ressalvar, entretanto, que os resultados estiveram condicionados a uma amostra pequena e a uma metodologia transversal. Dada a importância das aplicações práticas de estudos desta natureza, identifica-se a necessidade da continuidade de estudos sobre a felicidade e seus fatores explicativos, especialmente através de métodos quantitativos e longitudinais. Considerando-se os conceitos elucidados para a amostra deste estudo, torna-se relevante analisar, futuramente, do ponto de vista do próprio adolescente, os fatores que garantem o seu bem-estar subjetivo. Para isso, recomenda-se partir da proposta da Psicologia Positiva, que busca enfatizar as potencialidades do indivíduo. Desse modo, é necessário o investimento em estudos que se dediquem ao desenvolvimento de instrumentos que auxiliem a medir esse construto.

\section{Referências}

Abaid, J. L. W., Dell'Aglio, D. D., \& Koller, S. H. (2010). Preditores de sintomas depressivos em crianças e adolescentes institucionalizados. Universitas Psychologica, 9, 199-212.

Arteche, A. X., \& Bandeira, D. R. (2003). Bem-estar subjetivo: um estudo com adolescentes trabalhadores. Psico-USF, 8(2), 193201.

Assumpção Jr., F. B. (2008). A adolescência. Em F. B. Assumpção
Jr. (Org.), Psicopatologia evolutiva (pp. 187-219). Porto Alegre: Artmed.

Assumpção Jr., F. B., Kuczynski, E., Sprovieri, M. H., \& Aranha, E. M. G. (2000). Escala de avaliação de qualidade de vida: (AUQEI - Autoquestionnaire Qualité de Vie Enfant Imagé) validade e confiabilidade de uma escala para qualidade de vida em crianças de 4 a 12 anos. Arquivos de Neuro-Psiquiatria, 58(1), 119-127.

Assis S. G., Avanci J. Q., Silva C. M. F. P., Malaquias, J. V., Santos N. C., \& Oliveira R. V. C. (2003). A representação social do ser adolescente: um passo decisivo na promoção da saúde. Ciências e saúde coletiva, 8(3), 669-679.

Bardin, L. (2004). Análise de conteúdo. (L. A. Reto \& A. Pinheiro, Trad.). São Paulo: Martins Fontes. (Original publicado em 1979)

Estatuto da Criança e do Adolescente. (1990). Diário Oficial da União. Lei $n^{\circ} 8069$, de 13 de julho de 1990. Brasília, DF.

Cole, D. A., Tram, J. M., Martin, J. M., Hoffman, K. B., Ruiz, M. D., Jacquez, F. M., \& Maschman, T. L. (2002). Individual differences in the emergence of depressive symptoms in children and adolescents: a longitudinal investigation of parent and child reports. Journal of Abnormal Psychology, 111(1), 156-165.

Conselho Federal de Psicologia. (2000). Resolução para pesquisas com seres humanos. Resolução 016/2000. Brasília.

Conselho Nacional de Saúde. (1996). Resolução no 196, de 10 de outubro de 1996. Recuperado: 01 mar 2006. Disponível: http:// www.datasus.gov.br/conselho/resol96/RES19696.htm.

Dancey, C. P. (2006). Estatística sem matemática para psicologia (Lori Viali, Trad). Porto Alegre: Artmed.

Dela Coleta, J. A., \& Dela Coleta, M. F. (2006). Felicidade, bemestar subjetivo e comportamento acadêmico de estudantes universitários. Psicologia em estudo, 11(3), 533-539.

Dell'Aglio, D. D., \& Hutz, C. S. (2002) Estratégias de coping de crianças e adolescentes em eventos estressantes com pares e com adultos. Psicologia-USP 13(2), 203-225.

Dell'Aglio, D. D., Koller, S. H., \& Yunes, M. A. M. (Orgs.). (2006) Resiliência e Psicologia Positiva: interfaces do risco à proteção. São Paulo: Casa do Psicólogo.

Diener, E. (1984). Subjective well-being. Psychological Bulletin, 95, $542-575$

Diener, E. (1996). Subjective well-being. International Journal of Psychology, 31(3-4), 2320-2320.

Diener, E., Suh, E., \& Oishi, S. (1997). Recent findings on subjective well-being. Indian Journal of Clinical Psychology, 24, 25-41. 
Diener, E., Suh, E. M., Lucas, R. E., \& Smith, H. L. (1999). Subjective well-being: three decades of progress. Psychological Bulletin, 125, 276-302.

Diener, E., \& Lucas, R. E. (2000). Explaining differences in societal levels of happiness: relative standards need fulfillment, culture, and evaluation theory. Journal of Happiness Studies, 1, 41-78.

Ferreira, M. de C. T., \& Marturano, E. M. (2002). Ambiente familiar e os problemas do comportamento apresentados por crianças com baixo desempenho escolar. Psicologia: reflexão e crítica, 15(1), 35-44.

Giacomoni, C. H. (2002). Bem-estar subjetivo infantil: conceito de felicidade e construção de instrumentos para avaliação. Tese de Doutorado, Universidade Federal do Rio Grande do Sul, Porto Alegre, Rio Grande do Sul.

Giacomoni, C. H., \& Hutz, C. S. (2008). Escala multidimensional de satisfação de vida para crianças: estudos de construção e validação. Estudos de Psicologia, 25(1), 25-35.

Graziano, L. D. (2005). A felicidade revisitada: um estudo sobre bemestar subjetivo na visão da Psicologia Positiva. Tese de Doutorado, Instituto de Psicologia, Universidade de São Paulo, São Paulo.

Huebner, E. S., Drane, W., \& Valois, R. F. (2000). Levels and demographic correlates of adolescent life satisfaction reports. School Psychology International, 21(3), 281-292.

Lastória, L. A. C. N. (2003). Impasses éticos na educação hoje. Educação \& Sociedade, 24(83), 429-440.
Luthar, S. S., \& Zigler, E. (1991). Vulnerability and competence: a review of research on resilience in childhood. American Journal Orthopsychiatry, 61, 6-22.

Malpique, C., Lima, R. Q., Soeiro, D., \& Confraria, L. (2003). Risco psiquiátrico na puberdade adiantada. Revista Portuguesa de Psicossomática, 5(2), 21-35.

Narvaz, M. (2001). A transmissão transgeracional da violência. Insight, 118(11), 17-22.

Passareli, P. M., \& Silva, J. A. (2007). Psicologia positiva e o estudo do bem-estar positivo. Estudos de Psicologia, 24(4), 513-517.

Seligman, M. E. P., \& Csikszentmihalyi, N. A. (2000). Positive psychology: an introduction. American Psychologist, 55(1), 5-14.

Sheldon, K. M., \& King, L. (2001). Why Positive Psychology is necessary. American Psychologist, 56, 216-217.

Steinberg, L. (1999). Adolescence. Boston: McGraw-Hill.

Werner, E., \& Smith, R. (1998). Vulnerable but invincible: a longitudinal study of resilient children and youth. New York: McGraw Hill.

Yunes, M. A. M. (2003). Psicologia positiva e resiliência: o foco no indivíduo e na família. Psicologia em Estudo, 8, 75-84.

Zagury, T. (2003). Debatedores. Ciência e Saúde Coletiva, 8(3), 681689. 


\section{Sobre as autoras}

Síglia Pimentel Höher Camargo (sigliahoher@yahoo.com.br)

Psicóloga, mestre pelo Curso de Pós-Graduação em Psicologia do Desenvolvimento da Universidade Federal do Rio Grande do Sul (UFRGS), doutoranda do Programa de Pós-Graduação em Educação Especial, Educational Psychology Department, Texas A\&M University, USA. Endereço: 8320 Grassbur Rd., Bryan, Texas, USA, 77808.

Josiane Lieberknecht Wathier Abaid (josianelieb@yahoo.com.br)

Psicóloga, mestre e doutoranda pelo Curso de Pós-Graduação em Psicologia do Desenvolvimento da Universidade Federal do Rio Grande do Sul (UFRGS); docente do Curso de Psicologia do Centro Universitário Franciscano (UNIFRA, RS). Endereço: Rua Euclides da Cunha, n. 1880, bairro Dores, CEP 97090-000, Santa Maria/RS.

Claudia Hofheinz Giacomoni (giacomon@uol.com.br)

Psicóloga, doutora pelo Curso de Pós-Graduação em Psicologia do Desenvolvimento da UFRGS e docente do Curso de Psicologia e do Programa de Pós-Graduação em Psicologia da Universidade Federal de Santa Maria (UFSM). Endereço: Av. Presidente Vargas, 2163 apt 03 CEP: 97015-513, Santa Maria/RS.

\section{Endereço para correspondência com o editor:}

Rua Euclides da Cunha, n. 1880, bairro Dores, CEP 97090-000, Santa Maria/RS. Telefone: (55) 33073807 josianelieb@yahoo.com.br Esta pesquisa foi desenvolvida junto ao Laboratório de Estudos, Medidas e Avaliação em Psicologia (LEMAP) da UFSM com apoio financeiro da FAPERGS.

Agradecemos aos acadêmicos de Psicologia da UFSM vinculados ao LEMAP pelo auxílio na coleta e levantamento dos dados, bem como aos adolescentes que se dispuseram a participar deste estudo. 